九州大学学術情報リポジトリ

Kyushu University Institutional Repository

\title{
Risk Assessment of a Large Constellation of Satellites in Low-Earth Orbit
}

Matsushita, Yuri

Kyushu University

Yoshimura, Yasuhiro

Kyushu University

HANADA, Toshiya

Kyushu University

I taya, Yuki

SKY Perfect JSAT Corporation

他

ht tp://hdl. hand le. net/2324/4755253

出版情報: Transactions of the Japan Society for Aeronautical and Space Sciences, Aerospace Technology Japan. 20, pp. 10-15，2022-02-05. 日本航空宇宙学会

バージョン:

権利関係: ๑ 2022 The Japan Society for Aeronautical and Space Sciences 


\title{
Risk Assessment of a Large Constellation of Satellites in Low-Earth Orbit
}

\author{
By Yuri Matsushita, ${ }^{1)}$ Yasuhiro Yoshimura, ${ }^{1)}$ Toshiya HanadA, ${ }^{1)}$ Yuki Itaya, ${ }^{2)}$ and Tadanori FukUshimA ${ }^{2)}$ \\ ${ }^{1)}$ Kyushu University, Fukuoka, Japan \\ ${ }^{2)}$ SKY Perfect JSAT Corporation, Tokyo, Japan
}

(Received March 18th, 2021)

\begin{abstract}
This paper aims to assess the risk of mission termination for a large constellation of satellites in a low-Earth orbit. Many large constellations will be deployed to provide broadband network services using thousands of satellites. There is concern that such large constellations will have a serious impact on the long-term sustainability of outer space activities due to the rapid increase in population. First, therefore, the authors conducted an assessment under nominal activities (referred to as "business-as-usual") on the basis of a prediction by ESA's MASTER-2009 and NASA standard breakup model 2001 revision. The assessment found that nearly one catastrophic collision may happen in a large constellation, generating more than two million fragments as small as $1 \mathrm{~mm}$ in size. Second, the authors conducted a further assessment assuming a hypothetical collision of a satellite in a large constellation using the NASA standard breakup model and a spherical finite element model adopted in ESA's MASTER-2009. In consequence, another catastrophic collision may happen to a large constellation, generating approximately a half-million fragments as small as $1 \mathrm{~mm}$ in size. Therefore, such catastrophic collisions and resulting secondary collisions should be prevented for large constellations.
\end{abstract}

Key Words: Space Debris, Large Constellation, Risk Assessment, End-of-Life Support

\section{Introduction}

The deployment of large constellations has been causing a rapid increase in the number of satellites orbiting the Earth and posing a major threat against the safety of the space environment. It is estimated that by 2030 there will be three times as many satellites as in $2018,{ }^{1}{ }^{1}$ most of which are to be launched into Low-Earth Orbit (LEO). The high congestion in the LEO region has already been affecting some satellite operators. For example, in 2019, ESA needed to move the Aeolus satellite to avoid a collision with Starlink 44, one of the satellites in SpaceX's constellation. ${ }^{2)}$ This was the first time for ESA to conduct a collision avoidance maneuver against a satellite constellation. ESA has reported that the number of close approaches to the satellites has been increasing over the years, and that this trend is estimated to be accelerated as the number of satellite constellations increases. ${ }^{3)}$ The Inter-Agency Space Debris Coordination Committee (IADC) has stated that the conventional debris mitigation guidelines are not sufficient to manage upcoming constellations effectively. ${ }^{4)}$ Therefore, further efforts need to be made by constellation operators to protect their own constellations as well as the surrounding spacecraft. To address this issue, some space agencies and private companies including SKY Perfect JSAT Corporation are currently working on the research and development of active debris removal (ADR).

ADR is mainly designed for removing larger objects, such

(C) 2022 The Japan Society for Aeronautical and Space Sciences as defunct satellites or rocket upper-stages, and smaller objects such as fragmentation debris are not considered as targets. However, recent studies indicate that small and untracked objects can also cause serious problems to the orbital environment. Especially, debris with a diameter of $1 \mathrm{~mm}$ and $10 \mathrm{~cm}$ are generally untracked from ground observatories, but they still have enough potential to cause mission-ending damage once they collide with operational satellites. ${ }^{5)}$ On 23 August 2016, a small fragment collided with the ESA Sentinel 1A satellite. An investigation has revealed that the impactor had almost the same characteristics as an aluminum sphere only $5 \mathrm{~mm}$ in diameter. ${ }^{6}$ ) The output power of the solar array pointing in the direction of flight dropped by $280 \mathrm{~W}$ from a nominal $6000 \mathrm{~W}$, but fortunately this event had no effect on the satellite's routine operations, which continue normally. This event implies that even a tiny object may make mission execution difficult depending on the degree of damage, however. The number of objects larger than $1 \mathrm{~mm}$ is estimated to exceed 100 million, ${ }^{5)}$ which is much higher than trackable objects (approximately 23,000 objects). Operational satellites, including satellites in constellations, are vulnerable to those "invisible" objects since spacecraft can neither detect nor avoid such debris.

To assess the influence of such smaller objects as well as larger trackable objects, space debris environmental models, such as NASA's ORDEM 3.0 and ESA's MASTER-2009, are widely used. Both models can compute the space debris population including smaller particles as a function of collision flux, which represents the impact rate per unit time per unit area. The accumulated flux data indicates the expected 
number of collisions and the collision probability for a certain satellite throughout its lifetime. Therefore, these models have been chosen for the constellation assessment tool, and recently there has been increasing interest towards analysis including smaller objects. Radtke et al. ${ }^{7)}$ use MASTER-2009 to include space objects down to $3 \mathrm{~cm}$ in size into the evaluation of collision probability associated with the OneWeb satellite constellation, and also consider fragments generated by a hypothetical self-induced collision to assess the impact on the constellation itself. Le May et al. ${ }^{8}$ chose OneWeb and SpaceX constellations and evaluated the probability of collision with space objects larger than $3 \mathrm{~mm}$ in size.

This paper aims to assess the risk of mission termination for a large constellation of satellites in LEO. Following the report of Liou, ${ }^{5)}$ this paper assumes that debris with a diameter of $1 \mathrm{~mm}$ or larger still has enough potential to cause mission-ending damage. In addition, this paper focuses not only on the number of such collisions, but also on the number of resulting fragments to assess the risk of mission termination due to secondary collisions. First, this paper evaluates the collision flux for satellites in a large constellation under nominal activities (referred to as "business-as-usual") on the basis of a prediction by ESA's MASTER-2009. Then, this paper uses the NASA standard breakup model 2001 revision to estimate how many collisions might lead to fatal damage or make the mission execution difficult in terms of an energy-to-mass ratio, defined as the relative kinetic energy of the smaller object divided by the mass of the larger object. Second, this paper evaluates the collision flux for satellites in a large constellation after a hypothetical catastrophic collision. This evaluation also uses the NASA standard breakup model 2001 revision and a spherical finite element model adopted in ESA's MASTER-2009. Finally, the authors conclude that it is necessary to remove defunct satellites in constellations and fragmentation debris for the long-term sustainability of outer space activities.

\section{Large Constellation}

Figure 1 illustrates a large constellation of satellites at an altitude of $1200 \mathrm{~km}$, which is assessed in this study. The large constellation contains 1980 satellites, with 55 satellites evenly distributed in each of 36 orbital planes. All of the orbital planes are assumed to be circular at the same inclination, $87.9 \mathrm{deg}$. The ascending nodes of 36 orbital planes are distributed around the equator at intervals of $5.1 \mathrm{deg}$. At any given time, therefore, half of the satellites are going northward and the other half are going southward, as in the "street of coverage" constellation pattern.

Figure 2 illustrates each satellite's configuration in the constellation, a box-wing type with a main cuboid body and two flat solar array paddles. The volume of the main body is $1.3 \mathrm{~m}$ by $1.0 \mathrm{~m}$ by $1.0 \mathrm{~m}$, while the area of each solar array paddle is $1.0 \mathrm{~m}$ by $1.3 \mathrm{~m}$. The main body and the solar array paddles are $1.0 \mathrm{~m}$ apart. The mass of each satellite is assumed to be $147.7 \mathrm{~kg}$. Finally, the constellation assumes to

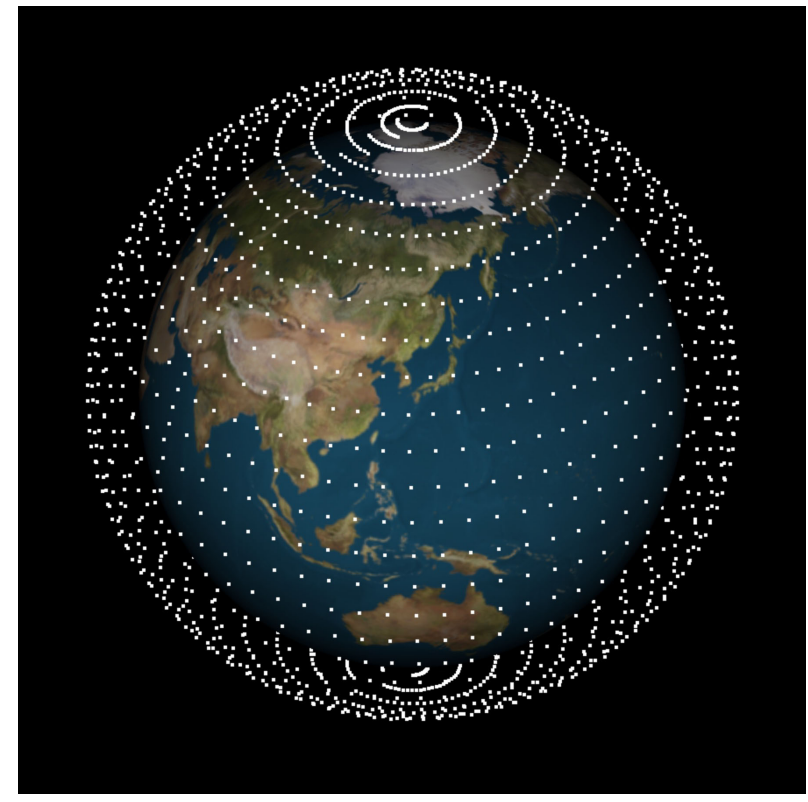

Fig. 1. Large constellation of satellites in low-Earth orbit.

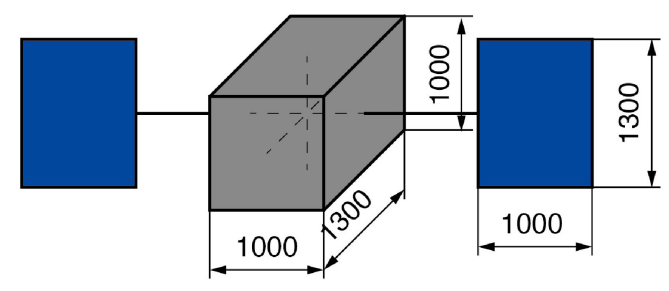

UNIT: $\mathrm{mm}$

Fig. 2. Configuration of satellites in the large constellation.

start its service at the beginning of 2020, with 1980 satellites fully deployed in the constellation from the beginning. Additionally, its service assumes to last for 30 years with 1980 satellites at all times, without consideration of replacements or spares.

\section{Risk Assessment under Nominal Activities}

As described in the Introduction, NASA's ORDEM and ESA's MASTER are widely used to assess the risk of mission termination for space missions. As also described in the Introduction, this paper focuses not only on the number of collisions that may cause mission termination, but also on the number of resulting fragments to assess the risk of mission termination due to secondary collisions. For this aim, the authors utilize ESA's MASTER instead of NASA's ORDEM because ESA's MASTER generates the cell passage event (CPE) dump files necessary for estimating the number of resulting fragments. Therefore, this section assesses the risk of the large constellation described in the previous section over the full-service duration of 30 years on the basis of a prediction made using ESA's MASTER-2009. ${ }^{9)}$ The latest version of ESA's MASTER-8 is currently available, but it is not utilized because its prediction is available until Novem- 


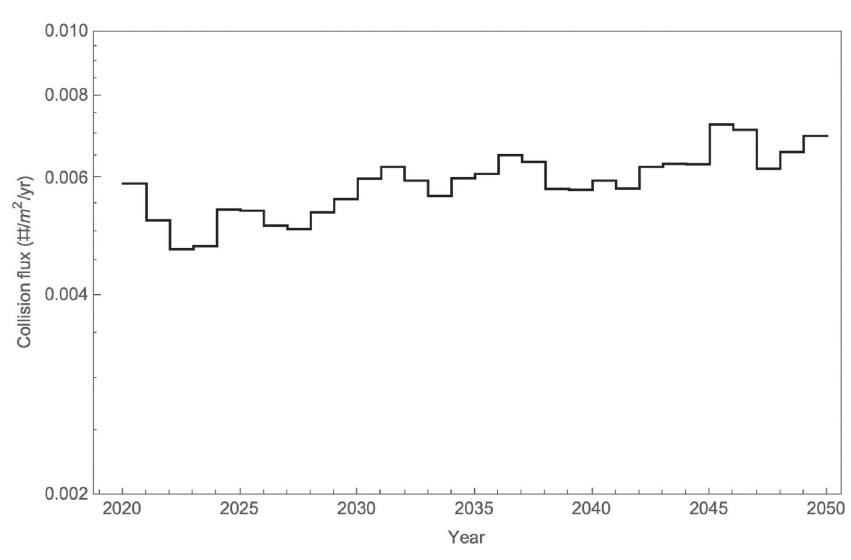

Fig. 3. Collision flux for the large constellation over the full-service duration.

ber $1,2036,{ }^{10)}$ not covering the full-service duration assumed. The prediction made using the previous version of ESA's MASTER-2009 is available until May 1, 2055, covering the full-service duration assumed.

The risk assessment is conducted under business-as-usual conditions. Business-as-usual is one of the future population evolution scenarios in ESA's MASTER-2009. In this scenario, explosion traffic and solid-rocket motor firing traffic are not reduced (to continue as usual), and mitigation measures are not implemented. This risk assessment also does not take into account fragments that may be released from the large constellation in comparison to the description provided in Section 4. As plotted in Fig. 3, the collision flux for the large constellation orbit over the full-service duration is approximately $6.0 \times 10^{-3}$ impacts $/ \mathrm{m}^{2} /$ year, with some fluctuation. Note that there is no significant difference in the prediction due to the difference in the ascending node, and Fig. 3 demonstrates the case that the ascending node is 0 deg.

The number of impacts that may happen to the large constellation can be estimated using Fig. 3. However, this study utilizes the NASA standard breakup model 2001 revision $^{11)}$ for the CPE dump files from ESA's MASTER2009 to estimate the number of fragments that the large constellation may release. The probability of collision with an object listed in the dump files can be evaluated as

$$
P_{\mathrm{col}}=\Phi A \Delta T
$$

where $\Phi$ is the individual flux contribution in the CPE dump files, $A$ is the effective cross-sectional area, and $\Delta T$ is the simulation duration. The effective cross-sectional area $A$ is expressed as

$$
A=\left(\frac{D_{\text {sat }}+D_{\text {obj }}}{2}\right)^{2}
$$

where $D_{\text {sat }}$ is the diameter of each satellite in the large constellation, and $D_{\text {obj }}$ is the diameter of the object that collides with the satellite, which is also provided with the CPE dump files. To calculate $D_{\text {sat }}$, this study only considers the main body that does not include appendages such as solar array panels, and its average cross-sectional area is assumed to be one quarter of the total surface area.

The NASA standard breakup model describes the number of fragments larger than a given characteristic length (in $\mathrm{m}$ ), $L_{C}$, as

$$
N_{\text {cum }}=0.1 M_{\text {tot }}^{0.75} L_{C}^{-1.71}
$$

where the value of $M_{\mathrm{tot}}$ is defined as the total mass (in $\mathrm{kg}$ ) of both objects in a catastrophic collision, where both objects are totally fragmented. In a non-catastrophic collision, primarily characterized by fragmentation of the smaller object and by cratering of the larger object, the value of $M_{\text {tot }}$ is defined as the product of the mass (in $\mathrm{kg}$ ) of the smaller object and the square of the collision velocity (in $\mathrm{km} / \mathrm{s}$ ) with a proportional factor of $1 \mathrm{~s}^{2} / \mathrm{km}^{2}$, giving the ejecta mass (in $\mathrm{kg}$ ). The smaller object is assumed to be fragmented in every collision and its mass is added to the ejecta mass. Note that the NASA standard breakup model defines the characteristic length as the average of three orthogonal projection dimensions, representing the size of fragments generated.

The NASA standard breakup model utilizes an energy-tomass ratio defined as the relative kinetic energy of the smaller object divided by the mass of the larger object. If the energy-to-mass ratio is equal to or greater than $40 \mathrm{~J} / \mathrm{g}$, then the collision is catastrophic. It should be known that the relative kinetic energy of the smaller object can be calculated as

$$
E=\frac{1}{2} m v_{\mathrm{rel}}^{2}
$$

where $m$ is the mass (in $\mathrm{kg}$ ) of the smaller object and $v_{\text {rel }}$ is the relative velocity (in $\mathrm{m} / \mathrm{s}$ ). The energy-to-mass ratio is thus given by

$$
E M R=\frac{E}{M}=\frac{1}{2} \frac{m}{M} v_{\text {rel }}^{2}
$$

where $M$ is the mass (in g) of the larger object. Figure 4 plots the energy-to-mass ratio of $40 \mathrm{~J} / \mathrm{g}$, which is the criterion for whether a collision is catastrophic or non-cata-

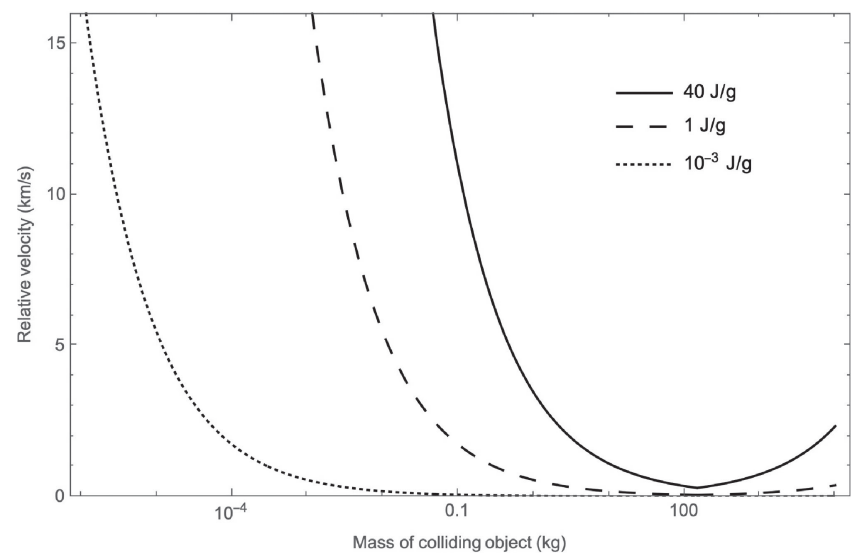

Fig. 4. Energy-to-mass ratio as a function of mass of colliding object and relative velocity. 
Trans. JSASS Aerospace Tech. Japan Vol. 20 (2022)

Table 1. The number of impacts and the number of fragments as expected values in terms of the energy-to-mass ratio.

\begin{tabular}{ccc}
\hline Energy-to-mass ratio $[\mathrm{J} / \mathrm{g}]$ & No. of impacts $[-]$ & No. of fragments $\left[\times 10^{6}\right]$ \\
\hline$\geq 0$ & 633.8 & 2.521 \\
$\geq 0.001$ & 351.0 & 2.516 \\
$\geq 1$ & 4.475 & 2.451 \\
$\geq 2$ & 3.166 & 2.442 \\
$\geq 5$ & 2.101 & 2.429 \\
$\geq 10$ & 1.567 & 2.416 \\
$\geq 20$ & 1.213 & 2.401 \\
$\geq 40$ & 0.9794 & 2.384 \\
\hline
\end{tabular}

strophic. Figure 4 also plots the energy-to-mass ratios of $1 \mathrm{~J} /$ $\mathrm{g}$ and $10^{-3} \mathrm{~J} / \mathrm{g}$. For satellites in the constellation, an energyto-mass ratio of $1 \mathrm{~J} / \mathrm{g}$ represents a head-on collision with a 1-cm-diameter aluminum sphere, and an energy-to-mass ratio of $10^{-3} \mathrm{~J} / \mathrm{g}$ represents a head-on collision with a $1-\mathrm{mm}$ diameter aluminum sphere. Therefore, a collision with the former ratio or higher may lead to fatal damage, and a collision with the latter ratio or higher may make mission execution difficult depending on the damage. Note that the satellite in the constellation is regarded as the larger object in calculating the energy-to-mass ratio when the mass of a colliding object is $147.7 \mathrm{~kg}$ or less, but the satellite in the constellation is regarded as the smaller object when the mass of a colliding object is $147.7 \mathrm{~kg}$ or more.

The number of fragments as an expected value can be calculated as the product of the probability of collision in Eq. (1) and the number of fragments in Eq. (2). Finally, the number of fragments that the large constellation may release over the full-service duration can be estimated by summing the number of fragments as expected for all individual objects in the CPE dump files. Table 1 summarizes the number of impacts and the number of fragments expected for some energy-to-mass ratios. Approximately 634 impacts with space debris larger than $1 \mathrm{~mm}$ in size may happen to the large constellation, resulting in approximately 2.5 million fragments also as small as $1 \mathrm{~mm}$ in size. Table 1 indicates that nearly one catastrophic collision may happen to the large constellation once. Furthermore, a few collisions may lead to fatal damage and approximately 350 collisions may make mission execution difficult. Therefore, a service satellite that removes defunct satellites in the constellation is essential to realizing and sustaining such a large constellation.

\section{Further Risk Assessment after a Hypothetical Cata- strophic Collision}

This section assumes a catastrophic collision of a satellite in the constellation, as estimated in the previous section, and then conducts a further risk assessment over the remaining service duration. The collision is assumed to take place at the beginning of 2030. The deployment of satellites in the constellation at the time of the collision is assumed to be the same as the initial deployment at the beginning of 2020 . Both right ascension of the ascending node and mean anom- aly of the satellite it collides with are assumed to be $0 \mathrm{deg}$. The colliding space debris mass is assumed to be $920.8 \mathrm{~kg}$ to generate 2.5 million fragments as small as $1 \mathrm{~mm}$ in size. This coincides with the number of fragments that the large constellation may release over the full-service duration, as can be seen from the data in Table 1. Of course, this study applies the size distribution for collision fragments using the NASA standard breakup model. It is almost impossible to uniquely determine the impact velocity, azimuth, and elevation of space debris involved in the collision, however. Therefore, this study applies the velocity distribution for explosion fragments in the NASA standard breakup model. Note that modifications that ESA's MASTER-2009 made to the NASA standard breakup model also apply.

To evaluate the collision flux of fragments generated, this study applies a spherical finite element model, which is also utilized in ESA's MASTER-2009. Outer space around the Earth is split into cells in terms of geocentric right ascension, declination, and distance. The dimension of a cell is set to $10 \mathrm{deg}$ in geocentric right ascension, $2 \mathrm{deg}$ in geocentric declination, and $10 \mathrm{~km}$ in geocentric distance. If a piece of fragment passes through a cell in which a satellite in the constellation also passes, then the satellite has a probability of colliding with the piece of fragment in the cell. The collision flux, $\Phi$, for a cell is calculated by

$$
\Phi=\frac{p p^{\prime} \Delta v}{V_{\text {cell }}}
$$

where $p$ and $p^{\prime}$ are the probabilities that the satellite and the piece of fragment will be in the cell, respectively, $\Delta v$ is the relative velocity between the satellite and the piece of fragment, and $V_{\text {cell }}$ is the volume of the cell containing both the satellite and the piece of fragment. The collision flux along the orbit can be calculated by summing up individual collision flux contributions evaluated in all cells through which the satellite passes.

Figure 5 compares the effective number of fragments for each altitude bin right after the collision (2030) and after the remaining service duration (2050). The size of the altitude bin is fixed to be $20 \mathrm{~km}$. The effective number below $640 \mathrm{~km}$ and above $1160 \mathrm{~km}$ decreases, but the effective number between $640 \mathrm{~km}$ and $1160 \mathrm{~km}$ increases. In other words, the orbital environment at altitudes of $640 \mathrm{~km}$ and $1160 \mathrm{~km}$ is worse than right after the collision. This altitude range includes sun-synchronous orbits that are useful for remote 


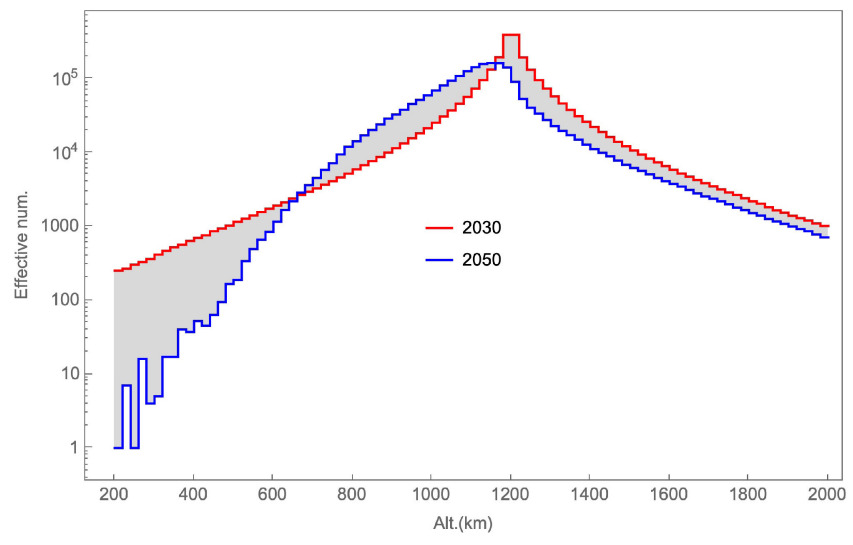

Fig. 5. Effective number of fragments for each altitude bin right after the collision (2030) and after the remaining service duration (2050).

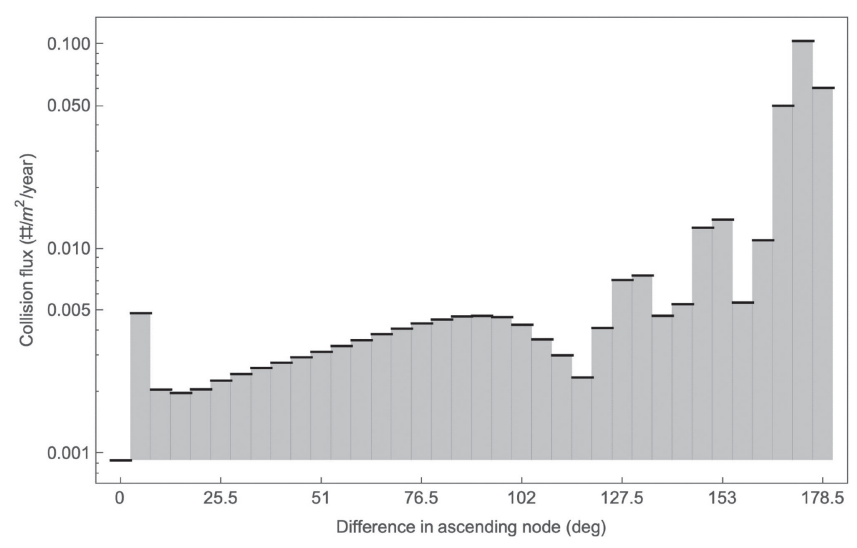

Fig. 6. Collision flux of fragments for each orbital plane right after the collision.

sensing. Accordingly, such collisions must be prevented for the long-term sustainability of outer space activities.

Figure 6 shows the collision flux of fragments for each orbital plane right after the collision. The collision flux of fragments ranges from $10^{-3}$ to $10^{-1}$ impacts $/ \mathrm{m}^{2} /$ year depending on the difference in the ascending node between the orbital plane where the satellite collision took place and each orbital plane. It can be seen that the collision flux of fragments for the same orbital plane where the satellite that collided with space debris is the smallest. The relative velocity at the intersections of two different orbital planes in the constellation increases as the difference in the ascending

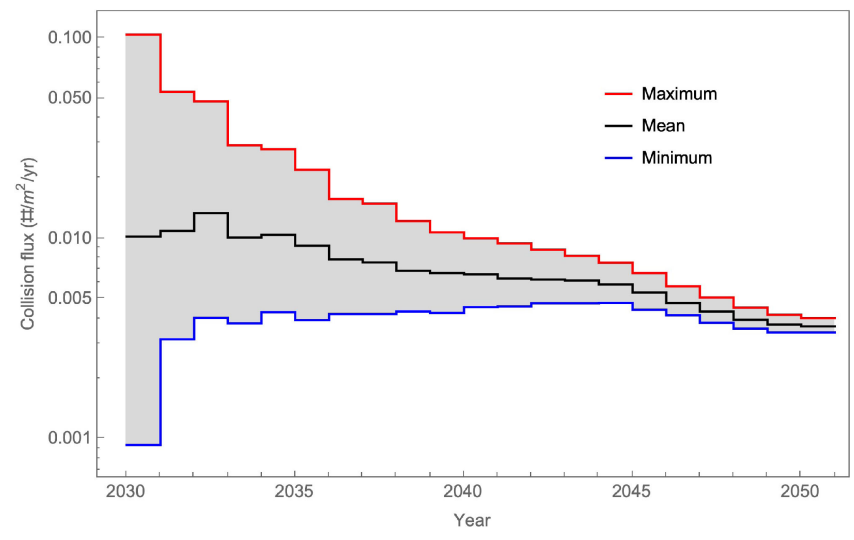

Fig. 7. Maximum, mean, and minimum collision flux of fragments over the remaining service duration.

node increases. Therefore, the larger the difference in the ascending node, the larger the collision flux of fragments tends to be.

Figure 7 plots the maximum, mean, and minimum collision flux of fragments over the remaining service duration. As can be seen from Fig. 7, the difference between the maximum and minimum collision flux is quite large right after the collision, but it gradually decreases. The maximum and minimum collision flux after the remaining service duration is almost the same as the mean collision flux. The nodal regression rate of each fragment is different, so that the orbital plane of each fragment deviates from the orbital plane where the collision takes place, and the fragments spread so as to cover the entire Earth. The collision flux difference, which is initially large due to the difference in the ascending node, gradually decreases.

Finally, Table 2 summarizes the number of impacts and the number of fragments generated as expected values over the remaining service duration. The hypothetical collision can cause 538 impacts in addition to the number of impacts estimated in the previous section. The resulting fragments can account for a half-million or more. Table 2 indicates another catastrophic collision may happen in the large constellation. Furthermore, it can be seen that there could be a few collisions that may lead to fatal damage and approximately 300 collisions that may make mission execution difficult.

Table 2. The number of impacts and the number of fragments as expected values in terms of the energy-to-mass ratio.

\begin{tabular}{ccc}
\hline Energy-to-mass ratio $[\mathrm{J} / \mathrm{g}]$ & No. of impacts $[-]$ & No. of fragments $\left[\times 10^{6}\right]$ \\
\hline$\geq 0$ & 536.1 & 0.5214 \\
$\geq 0.001$ & 299.2 & 0.5175 \\
$\geq 1$ & 3.264 & 0.4690 \\
$\geq 2$ & 2.448 & 0.4633 \\
$\geq 5$ & 1.650 & 0.4531 \\
$\geq 10$ & 1.190 & 0.4422 \\
$\geq 20$ & 0.7851 & 0.4262 \\
$\geq 40$ & 0.5104 & 0.4081 \\
\hline
\end{tabular}




\section{Conclusion}

The authors of this paper conducted a risk assessment when a large constellation consisting of 1980 satellites at an altitude of $1200 \mathrm{~km}$ continues service for 30 years. First, this assessment was conducted under nominal activities on the basis of a prediction using ESA's MASTER-2009. The use of the fragment size distribution function and the definition of catastrophic collisions in the NASA standard breakup model 2001 revision found that nearly one catastrophic collision may take place in the large constellation, resulting in 2.5 million fragments as small as $1 \mathrm{~mm}$ in size. Therefore, the authors continued a further risk assessment after a hypothetical catastrophic collision that generates 2.5 million fragments. This further assessment was done using the NASA standard breakup model and a spherical finite element model in ESA's MASTER-2009. It was found that another catastrophic collision may take place in the large constellation. In addition to these catastrophic collisions, these assessments also found that several collisions may lead to fatal damage and nearly 650 collisions may make mission execution difficult. Therefore, prevention of such a catastrophic collision and resulting secondary collisions is essential to realizing and sustaining such a large constellation.

\section{Acknowledgments}

The authors acknowledge the delegated work done by Mr. Daigo Izumizawa, Mr. Daisuke Sakai, and Mr. Shuta Fukii, graduate and undergraduate students at Kyushu University, on their calculation and data analysis.

\section{References}

1) Barnhart, D.: CONFERS; Fostering Standards to Enable Commercial Satellite Servicing, Proceedings of the International Symposium on Ensuring Stable Use of Outer Space, Tokyo, Japan, 28 February-1 March 2019.

2) Wall, M.: European Satellite Dodges Potential Collision with SpaceX Starlink Craft, 2019, https://www.space.com/spacex-starlink-esasatellite-collision-avoidance.html (accessed 2021.02.22).

3) ESA: ESA Environmental Report on Space Debris Mitigation, 2019, https://www.sdo.esoc.esa.int/environment_report/ (accessed 2021.02. 22).

4) IADC: IADC Statement on Large Constellations of Satellites in Low Earth Orbit, 2017 https:/www.iadc-home.org/documents_public/ file_down/id/4195 (accessed 2021.02.22).

5) Liou, J.-C.: Orbital Debris Mitigation in Support of Space Situational Awareness and Space Traffic Management, Proceedings of the International Symposium on Ensuring Stable Use of Outer Space, Tokyo, Japan, 28 February-1 March 2019.

6) Krag, H., Serrano, M., Braun, V., Kuchynka, P., Catania, M., Siminski, J., Schimmerohn, M., Marc, X., Kuijper, D., Shurmer, I., O'Connell, A., Otten, M., Muñoz, I., Morales, J., Wermuth, M., and McKissock, D.: A $1 \mathrm{~cm}$ Space Debris Impact onto the Sentinel-1A Solar Array, Acta Astronautica, 137 (2017), pp. 434-443.

7) Radtke, J., Kebschull, C., and Stoll, E.: Interactions of the Space Debris Environment with Mega Constellations Using the Example of the OneWeb Constellation, Acta Astronautica, 131 (2017), pp. 55-68.

8) Le May, S., Gehly, S., Carter, B. A., and Flegel, S.: Space Debris Collision Probability Analysis for Proposed Global Broadband Constellations, Acta Astronautica, 151 (2018), pp. 445-455.

9) Flegel, S.: Maintenance of the ESA MASTER Model, Final Report 21705/08/D/HK, Institute of Aerospace Systems, TU Braunschweig, 2011.

10) Braun, V., Horstmann, A., Lemmens, S., Wiedemann, C., and Bottcher, L.: Recent Developments in Space Debris Environment Modelling, Verification and Validation with MASTER, Proceedings of the 8th European Conference on Space Debris (virtual), Darmstadt, Germany, April 2-23, 2021, published by the ESA Space Debris Office, Flohrer, T., Lemmens, S., and Schmitz, F., eds. (http:// conference.sdo.esoc.esa.int, May 2021).

11) Johnson, N. L., Krisko, P. H., Liou, J.-C., and Anz-Meador, P. D.: NASA's New Breakup Model of EVOLVE 4.0, Adv. Space Res., 28, 9 (2001), pp. 1377-1384. 